\title{
Sensitivity Analysis of a Local Market Model for Community Microgrids
}

\author{
Laurine Duchesne, Bertrand Cornélusse \\ Department of EE \& CS \\ Montefiore Institute \\ University of Liège \\ Liège, Belgium \\ \{1.duchesne, bertrand.cornelusse\}@uliege.be
}

\author{
Iacopo Savelli \\ Dipartimento di Ingegneria \\ dell'Informazione e Scienze Matematiche \\ Università di Siena \\ Siena, Italy \\ savelli@diism.unisi.it
}

\begin{abstract}
A community microgrid is a microgrid composed of several entities, or members, that can share energy among themselves. The members of the community can match their demand and supply through an internal local market with a significant reduction of the exchanges with the main grid. As a consequence each participant can benefit from a reduction of its energy costs when the energy available locally is cheaper than the energy from the grid, from a drop of the energy peak demanded from the main grid, and from the new capability to provide energy reserve at aggregate level. In this paper, we analyze how the changes of the community market model parameters can affect both the community as a whole, and the welfare of each participant. The analysis is performed by varying the main drivers of the community market model, the community and storage fees, and the storage capacity. The numerical results are obtained by using real data based on the MeryGrid project.

Index Terms-community market, community microgrid, energy market, marginal pricing, sharing economy.
\end{abstract}

\section{INTRODUCTION}

The increasing share of renewable energy sources and storage systems in distribution grids opens the possibility for new market models that favor a local usage of the generated electricity. Local energy communities, and more specifically community microgrids, constitute one of these options. A microgrid is a set of loads, generators and storage devices connected by an electric grid within a clearly defined neighborhood, able to work either connected or disconnected from the main grid. It becomes a community microgrid when several legal entities constitute the microgrid, e.g. a set of small and medium-sized enterprises.

In the literature, microgrids have been explored under different aspects, however references related to community market models are limited. Reference [1] shows how to model and price co-generated energy within a local heat district, managed by a monopolistic public utility. Reference [2] proposes a microgrid model with internal exchanges formalized as a Nash bargaining problem, where the community prices are restricted to predetermined, discrete price levels. Reference [3] describes a community model where the participating units act in a collaborative manner. The optimal solution is obtained through a distributed algorithm by exploiting the alternating direction method of multipliers. Reference [4] presents a blockchainbased microgrid based on a pilot project built in Brooklyn, where blockchains appear to be an eligible technology to manage local microgrids. Reference [5] reviews and analyzes the most important market architectures for community microgrid, including decentralized peer-to-peer structures with direct trades among participants, and local community of aggregated units either connected to the main grid or islanded. Reference [6] proposes a peer-to-peer microgrid model where the internal community prices are determined heuristically depending on the ratio between the energy supplied and demanded within the community. Reference [7] describes a novel market model for community microgrids that is formalized as a bilevel problem. By using the proposed architecture, the community participants can allocate efficiently their resources with a significant reduction of the energy costs. Furthermore, the entities can pool their resources to provide ancillary services to the main grid. Moreover, by exploiting the netting effects at aggregate level, they can reduce the energy imported from the main grid, with a considerable drop in the energy peak costs. By using predetermined profit and cost sharing policies, a community operator ensures no participating entity is penalized.

The aim of this paper is to perform an in-depth sensitivity analysis, by measuring the effects of changing the main parameters of the community microgrid market model introduced in [7], in order to assess the soundness, reliability, and robustness of the proposed market architecture. In particular, we focus on the change of welfare for

- the community and community members,

- the storage owners,

- the community operator.

The analysis is performed by varying the following parameters:

- the fee collected by the storage owner,

- the fee collected by the community operator to manage the community,

- the amount of storage capacity available.

The remaining part of this paper is organized as follows. Section II briefly summarizes the model proposed in [7]. Section III describes the analysis performed and reports the numerical results. Finally, Section IV outlines the main conclusions. 


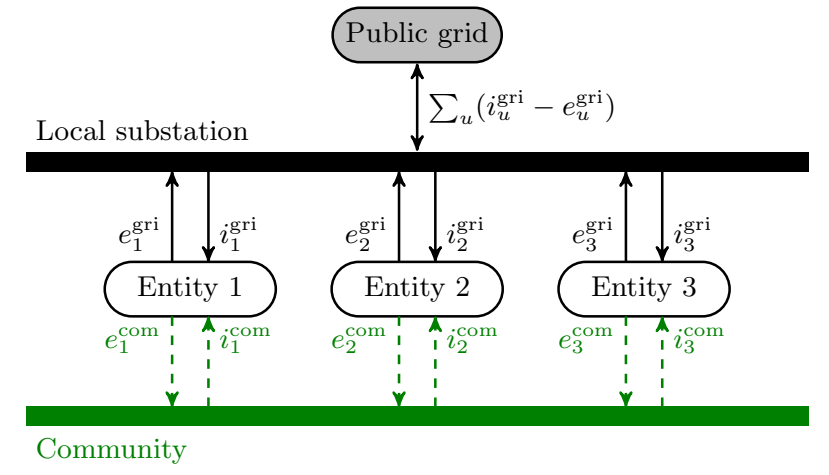

Fig. 1. Schematic community model. The flow $e_{i}^{\text {com }}$ (resp. $i_{i}^{\text {com }}$ ) represents the export (resp. import) of entity $i$ to the community. Flows tagged by "gri" denote traditional exchanges with the public grid.

\section{MOdel Summary}

This section briefly summarizes the market model for community microgrid described in [7]. The proposed architecture considers the entities forming the community, and a community operator that manages the community in the best interest of the participants. Collectively, the entities decide both the quantity to trade among themselves within the community, and the quantity to trade outside of the community directly with the main grid. This is illustrated in Fig. 1.

The market clearing at community level is performed by matching the demand and supply orders of each entity through a social welfare maximization problem. As a result, a market equilibrium is determined, where the executed quantity and the market prices are identified. By using the community, the entities can achieve a more efficient allocation of the resources, and therefore a reduction of the energy costs. Furthermore, as a group, the entities are able to provide energy reserve to the main grid and are able to effectively reduce the community energy peak, due to the import/export netting effect at aggregate level. Notice however that both the reserve provision and the community peak reduction are results of a collective effort. Therefore, some policy rules, and an independent operator (the community operator) are needed in order to determine how these benefits should be shared among the participating entities. Thus, given the market clearing results, a community operator must apply predetermined sharing policies in order to split the collective revenues and costs among each participant. An operator fee is considered to remunerate the activity of the community operator ${ }^{1}$. Similarly, storage device owners collect a battery fee each time their devices are used.

A bilevel model has been implemented in order to model this architecture. A bilevel model consists of two nested optimization problems, termed upper and lower level problems

\footnotetext{
${ }^{1}$ Note that it is assumed that the community operator has a contract with the DSO, since the community exploits the public grid for the physical flows. Therefore, the operator fee is used not only to remunerate the operator but also the DSO.
}

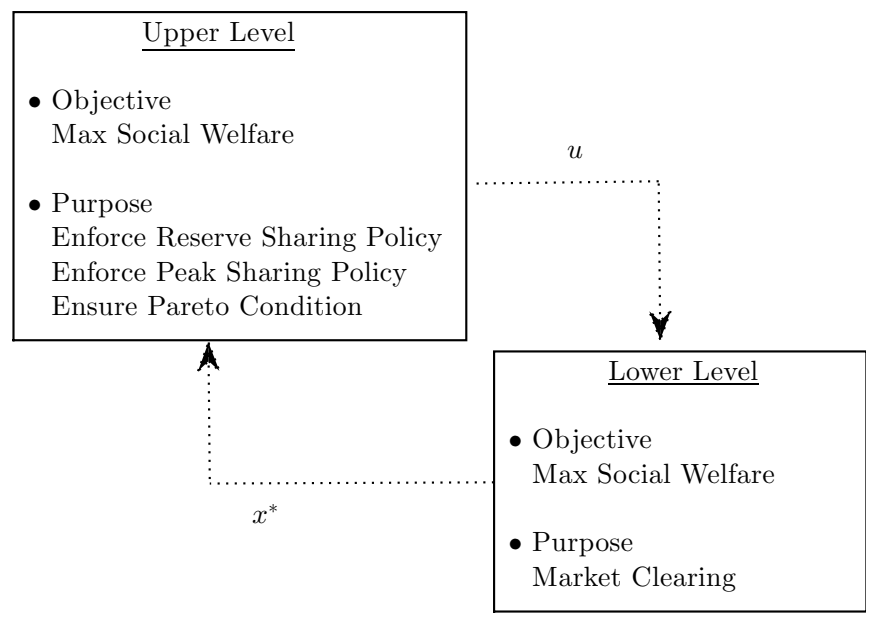

Fig. 2. The bilevel model structure. The lower level problem determines both the market equilibrium within the community, and the exchanges with the main grid. The upper level problem represents the community operator.

[8]. This structure can be sketched as follows:

$$
\begin{aligned}
& \max _{u \in \mathcal{U}} F\left(u, x^{*}\right) \\
& \text { s.t. } x^{*} \in \arg \max _{x \in \mathcal{X}} f(x ; u),
\end{aligned}
$$

where $F$ is the objective function of the upper level, and $f$ is the objective function of the lower level. In the proposed community microgrid model with internal market, the lower level part clears the community market by determining the executed quantities and the market prices, whereas the upper level acts as an independent community operator that applies the sharing policies, and ensures that no entity is penalized by participating in the community. The latter requirement is termed Pareto superior condition, and it is a fundamental condition in order to ensure a participation on a voluntary basis. Monetary compensations within the community are allowed. The overall process is sketched in Fig. 2.

A key feature of the proposed model is that the lower level problem is a linear program. By exploiting this characteristic, the lower level can be directly represented within the upper level by using its first order Karush-Kuhn-Tucker conditions, obtaining a single optimization problem equivalent to the initial bilevel model.

\section{Sensitivity Analysis}

In this section, we perform a sensitivity analysis of the proposed bilevel model on the following parameters: the community operator fee, the storage owner fee, and the capacity of the storage system. The purpose is two-fold: first to assess how different values of the parameters can affect the community and each participant; second to find a good value for each of these parameters.

We conduct this study on a realistic test case inspired by the MeryGrid project [9]. The MeryGrid project is a community microgrid composed of four entities. The first entity is a pure consumer. Entities 2 and 3 are both consumers and producers, 
TABLE I

MEAN $(\mu)$, STANDARD DEVIATION $(\sigma)$, AND MAXIMUM VALUES OF THE CONSUMPTION AND RENEWABLE GENERATION OF EACH ENTITY AND THE COMMUNITY RESPECTIVELY IN JANUARY AND IN AUGUST, IN KW.

\begin{tabular}{|rl|rrr|rrr|}
\cline { 3 - 7 } \multicolumn{1}{l|}{} & \multicolumn{3}{c|}{ January } & \multicolumn{3}{c|}{ August } \\
\cline { 1 - 7 } Entity & Type & $\mu$ & $\sigma$ & Max & $\mu$ & $\sigma$ & Max \\
\hline 1 & Load & 27 & 30 & 237 & 23 & 29 & 164 \\
2 & Load & 39 & 22 & 91 & 7 & 10 & 47 \\
2 & PV gen. & 0 & 1 & 26 & 5 & 11 & 67 \\
3 & Load & 21 & 37 & 183 & 17 & 36 & 193 \\
3 & Hydro gen. & 45 & 37 & 116 & 54 & 51 & 183 \\
\hline \hline Total & Load & 87 & 61 & 417 & 47 & 58 & 320 \\
& Generation & 45 & 37 & 123 & 59 & 54 & 224 \\
\hline
\end{tabular}

with respectively $70 \mathrm{kWp}$ of $\mathrm{PV}$ and $200 \mathrm{kVA}$ of hydro-electric capacity. The last entity, entity 4 , is a battery storage system with a capacity of $270 \mathrm{kWh}$. We use as demand and supply orders for the model the consumption and renewable generation of the four entities that were measured every 15 minutes during the year 2017. We focus on two months, January and August, chosen to evaluate the impact of some parameters with different weather conditions. Table I summarizes the consumption and generation data for these two months. On average, the consumption is larger in January than in August, but it is the contrary for the generation.

We consider as fixed the parameters depending on the contract with the main grid: the peak cost and the prices at which each entity can buy from and sell to the grid. Their values are respectively $0.15 €$ per $\mathrm{kW}, 0.15 €$ per $\mathrm{kWh}$ and $0.035 €$ per $\mathrm{kWh}$. Concerning the battery storage system, we assume that the charging and discharging efficiencies are both equal to 0.95 . Furthermore, the initial and final state of charge of the battery is equal to half its capacity. One instance of the problem lasts one day with time steps of 15 minutes. We do not consider reserves in our test case.

\section{A. Impact of the community operator fee}

The operator fee $\gamma^{\text {com }}$ is collected by the community operator each time an entity buys from or sells to another entity in the community. In this study, the value of $\gamma^{c o m}$ varies from 0.005 to $0.10 € / \mathrm{kWh}$ with steps of 0.005 . All the other parameters stay constant, in particular the storage owner fee is equal to $0.04 € / \mathrm{kWh}$.

Fig. 3 shows the total fees collected by the community operator during January 2017 as a function of the operator fee per $\mathrm{kWh}$. The operator revenue increases almost linearly and reaches the maximum with a tariff of $0.055 € / \mathrm{kWh}$. With a larger $\gamma^{\mathrm{com}}$, no fee is collected, meaning that there are no exchanges within the community. This analysis shows that from the community operator's point of view, the operator fee should be $0.055 € / \mathrm{kWh}$ to maximize his revenue.

To check if the value of $0.055 € / \mathrm{kWh}$ is meaningful, note that it is interesting for an entity to sell to the community

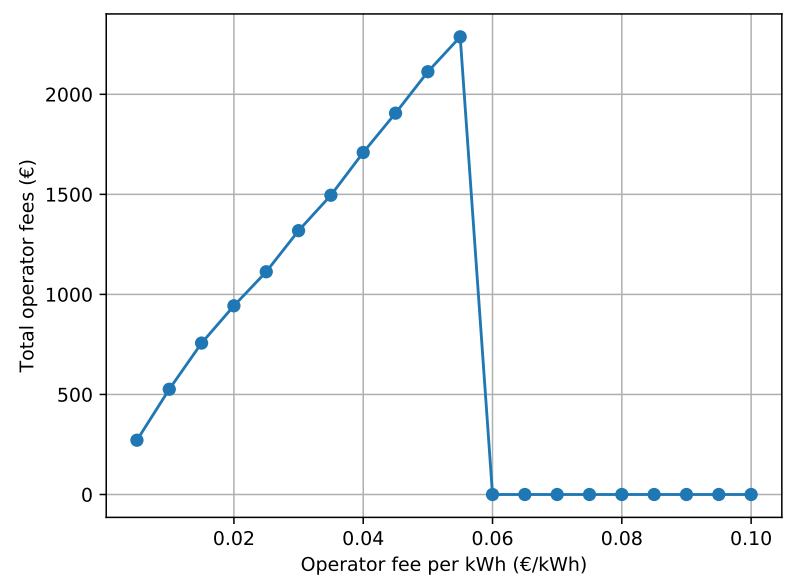

Fig. 3. Total fees paid to the operator during January 2017 as a function of $\gamma^{\text {com }}$.

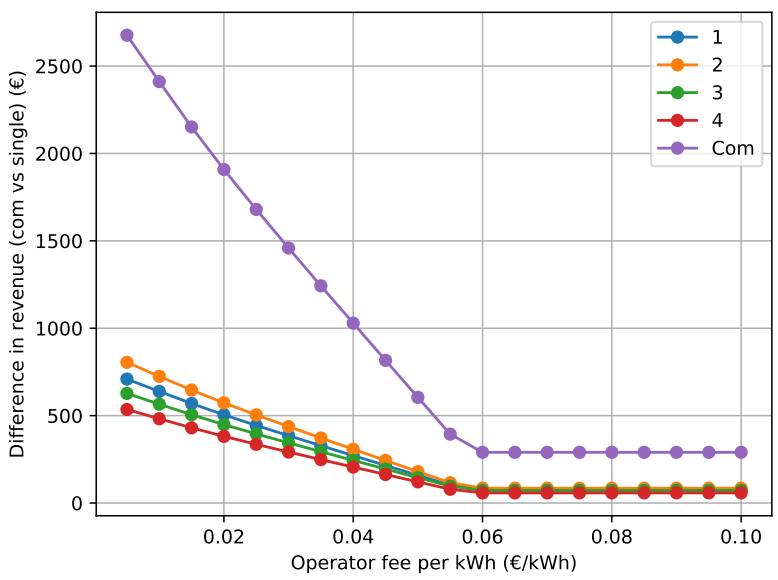

Fig. 4. Difference in revenue for being in the community compared to being a single entity during January 2017 as a function of $\gamma^{\mathrm{com}}$.

instead of the grid only if the community market price is equal to or larger than the grid purchase price. Thus, the minimum selling price to the community for an entity is 0.035 $€ / \mathrm{kWh}$. In that case, the buying entity has to pay this price and remunerate the community operator. The selling entity must also remunerate the community operator. However, in the proposed framework, the selling entity charges this cost to the buying entity. Therefore, the latter pays a minimum of $0.035+2 \times \gamma^{\text {com }} € / \mathrm{kWh}$. If this quantity is greater than $0.15 € / \mathrm{kWh}$ (the grid selling price), that is if $\gamma^{\mathrm{com}}$ is greater than $0.05825 € / \mathrm{kWh}$, no entity is willing to buy from the community, and therefore the selling entity sells to the grid. This explains the zero revenue for the community operator for $\gamma^{\text {com }} \geq 0.06 € / \mathrm{kWh}$. Even though there is no exchange between entities, Fig. 4 shows that it is still profitable for each entity to stay in the community, since 


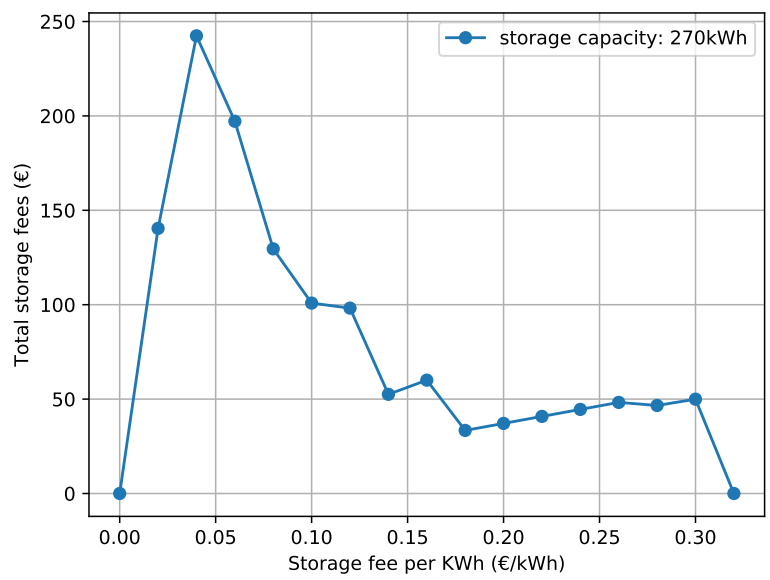

Fig. 5. Total storage fees during January 2017 as a function of $\gamma^{\text {sto }}$ for a storage capacity of $270 \mathrm{kWh}$.

joining the community decreases the peak penalty for the members. In particular, each entity improves its condition, and the Pareto condition is thus met. However, adopting a peak penalty scheme at the community level will likely force the distribution system operator (DSO) to increase the peak penalty tariff, which should incentivize community members to decrease and desynchronize their peak consumptions.

\section{B. Impact of the storage fee}

A storage fee per $\mathrm{kWh} \gamma^{\text {sto }}$ is collected by the battery owner each time the battery is charged or discharged. In this work $\gamma^{\text {sto }}$ varies from 0 to $0.32 € / \mathrm{kWh}$ with steps of 0.02 . Furthermore, the community operator fee is equal to 0.01 $€ / \mathrm{kWh}$.

Fig. 5 shows how the total fees collected by the storage owner in January evolves with the storage fee per $\mathrm{kWh}$. A first interesting observation is that it reaches the maximum value with $\gamma^{\text {sto }}=0.04 € / \mathrm{kWh}$. It is therefore a good choice for the battery owner to pick this value as storage fee, for the nominal capacity of the storage system.

After reaching the maximum, the fees collected by the storage owner do not decrease monotonically. We can explain this by looking at the use of the battery (the sum of charge and discharge actions). Fig. 6 shows that it decreases exponentially as a function of the storage fee per $\mathrm{kWh}$. Therefore, for large values of $\gamma^{\text {sto }}$, the decrease in the use of the battery does not compensate the increase of $\gamma^{\text {sto }}$, explaining why the total storage fees collected by the storage owner increase with $\gamma^{\text {sto }}$.

We notice that the storage system is not exploited anymore if $\gamma^{\text {sto }} \geq 0.32 € / \mathrm{kWh}$. In fact, it is shown in [7, (39)] that the community market price $\pi_{4, t+n}^{c o m}$ for entity 4 at discharging time $t+n$, is defined as

$$
\pi_{4, t+n}^{c o m}=\frac{\pi_{4, t}^{c o m}}{\eta^{c h a} \eta^{d i s}}+\frac{2 \gamma^{\text {sto }}}{\eta^{d i s}},
$$

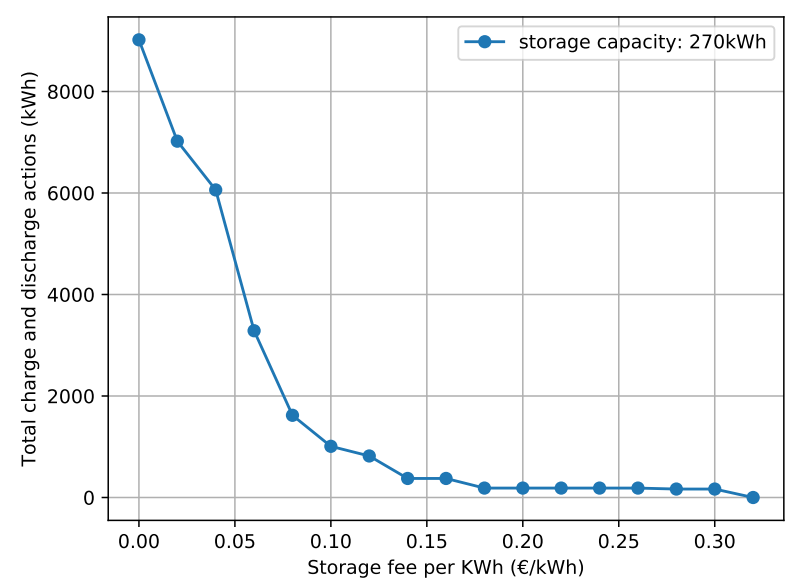

Fig. 6. Total charge and discharge actions during January 2017 as a function of $\gamma^{\text {sto }}$ for a storage capacity of $270 \mathrm{kWh}$.

where $\eta^{c h a}$ and $\eta^{\text {dis }}$ are the charging and discharging efficiencies of the battery, and $\pi_{4, t}^{c o m}$ is the market price paid by entity 4 at time $t$ to charge the battery. This market price is such that it compensates for the price paid by the battery owner to charge the battery, and for the losses due to the charging and discharging efficiencies. It also considers the storage fee. Notice that, the maximum market price an entity $d$ is willing to pay, in order to buy electricity within the community, is capped by the price paid to buy directly from the grid. In this setting, this value is equal to $0.75 € / \mathrm{kWh}^{2}$, and it is given by the following relation [7]

$$
\pi_{d}^{i g r}+\frac{\pi_{p e a k}}{\Delta_{T}}=0.75 € / \mathrm{kWh}
$$

with the grid selling price $\pi_{d}^{i g r}=0.15 € / \mathrm{kWh}$, the peak price $\pi_{\text {peak }}=0.15 € / \mathrm{kW}$ and $\Delta_{T}=0.25$ hours. It is therefore necessary for the market price of entity 4 at discharging time, i.e. $\pi_{4, t+n}^{c o m}$ in (3), to be smaller than $0.75 € / \mathrm{kWh}$ to be attractive. Assuming a charging price $\pi_{4, t}^{c o m}$ equal to its minimum value, $0.035 € / \mathrm{kWh}$, this condition is fulfilled only if $\gamma^{\text {sto }}<0.3378$, which explains the values observed in both Fig. 5 and Fig. 6.

\section{Impact of the storage fee and the capacity of the storage system}

We now study simultaneously the impact of the storage fee per kWh and the capacity of the storage system. For this analysis, the storage fee per kWh varies as in Section III-B and the storage capacity varies from 0 to $540 \mathrm{kWh}$ with steps of $30 \mathrm{kWh}$.

Fig. 7 shows the total fees collected by the battery owner in January and in August as a function of the capacity of the battery, for different values of $\gamma^{\text {sto }}$. The capacity of the battery

\footnotetext{
${ }^{2}$ Note that this situation occurs only when all entities need to import from the grid and therefore the battery is used to reduce the maximum peak value by spreading the import over several time steps.
} 


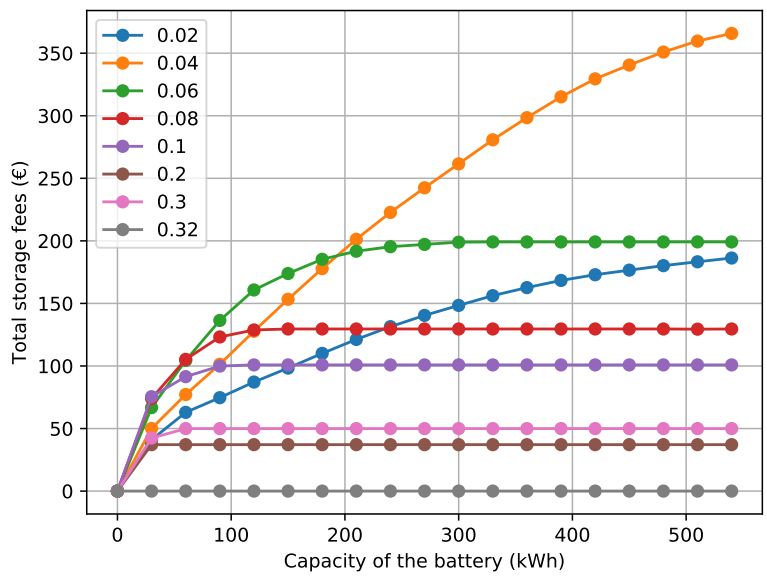

(a) January

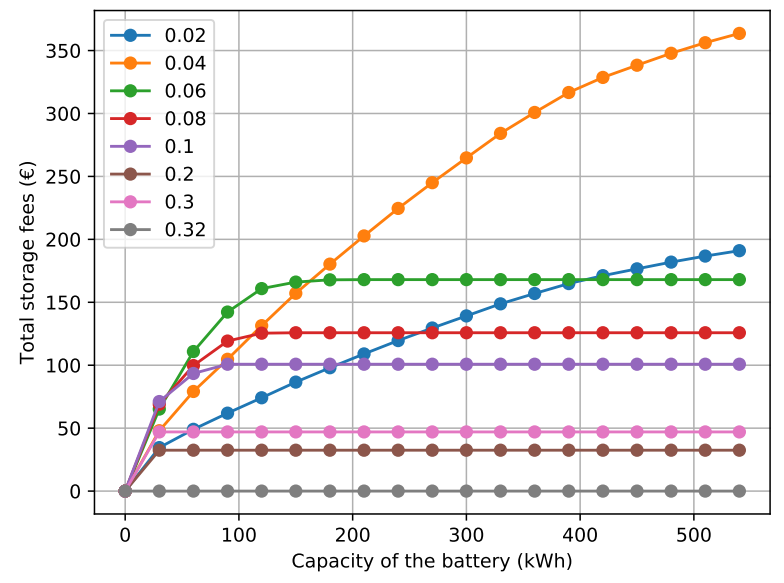

(b) August

Fig. 7. Total storage fees as a function of the storage capacity for various $\gamma^{\text {sto }}$, respectively in January and in August.

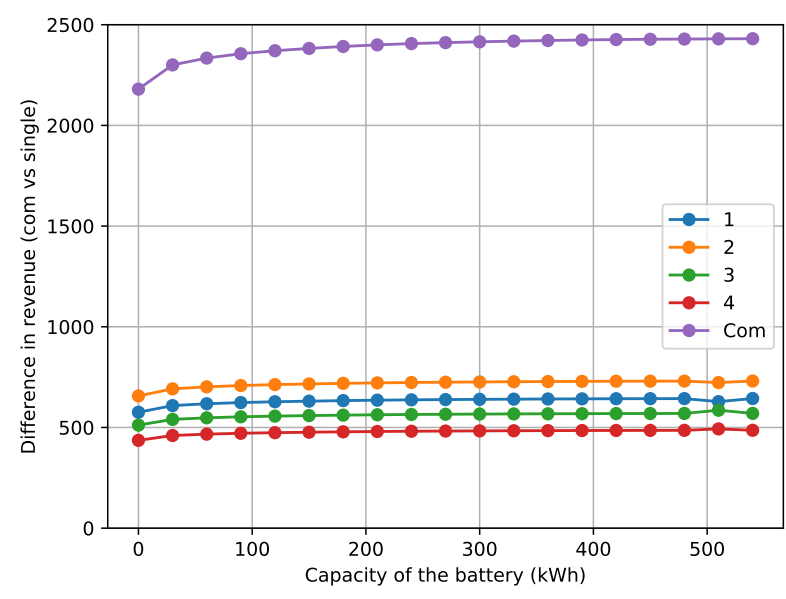

(a) January

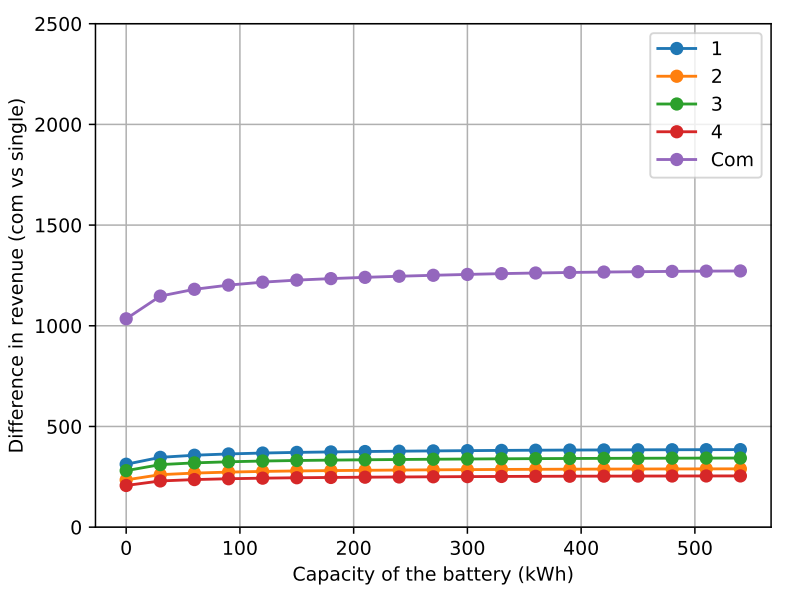

(b) August

Fig. 8. Difference in revenue for being in the community compared to being a single entity as a function of the storage capacity for $\gamma^{\text {sto }}=0.04 € / \mathrm{kWh}$, respectively in January and in August.

maximizing the owner revenue is very sensitive to the value $\gamma^{\text {sto }}$. For a large $\gamma^{\text {sto }}$, the current capacity of the community $(270 \mathrm{kWh})$ is too large. This graph corroborates the observation made in the previous subsection: with $\gamma^{\text {sto }}=0.32$, the battery is not used, independently of its capacity.

For both January and August, despite the difference in consumption and generation profiles, the value of 0.04 for the storage fee per $\mathrm{kWh}$ leads to the maximum revenue for the storage owner, if the storage capacity is large enough. Otherwise, there is a clear dependency between the capacity of the storage unit and the $\gamma^{\text {sto }}$ maximizing the owner fees.

Fig. 8 show the difference in revenue for being in the community compared to being a single entity as a function of the storage capacity, for $\gamma^{\text {sto }}=0.04 € / \mathrm{kWh}$. The difference is positive for all entities, due to the Pareto condition. The difference in revenue for being in the community increases similarly for all entities when the capacity increases.

The opposite behavior can be observed in Fig. 9 which also shows the difference in revenue for being in the community, but this time as a function of $\gamma^{\text {sto }}$.

In both figures, we notice a clear difference between January and August. It seems that it is more interesting to be in the community in January, when the consumption is higher than the production in average.

We see that the entities that make the most of the community are entity 2 in January and entity 1 in August. They are the largest consumers in average for the given month and they seem to exploit well the production surplus of the other entities. 


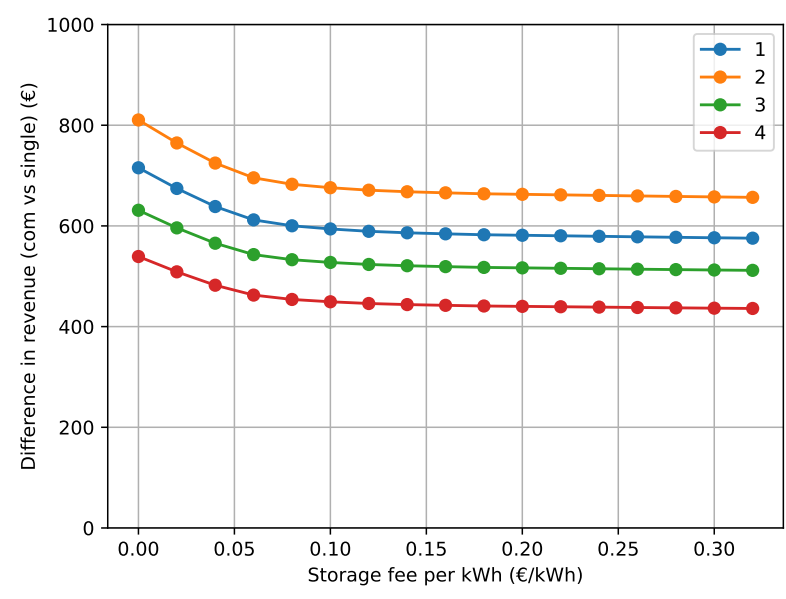

(a) January

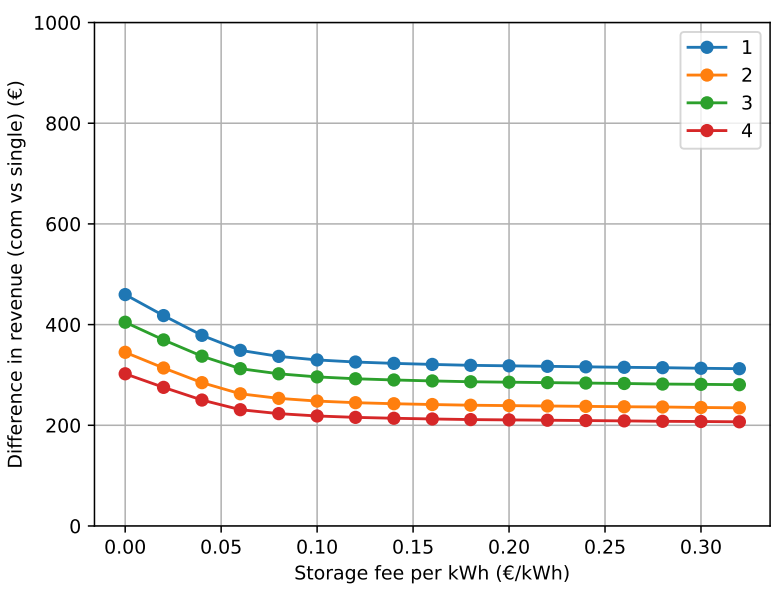

(b) August

Fig. 9. Difference in revenue for being in the community compared to being a single entity as a function of $\gamma^{\text {sto }}$, and for the nominal storage capacity $(270 \mathrm{kWh})$, respectively in January and in August.

\section{CONCLUSION}

Community microgrids represent an emerging topic in energy systems. These new architectures allow participants to exchange energy and resources among themselves, and to share the resulting benefits.

By using a novel community microgrid market model, the proposed sensitivity analysis helps to shed light on how different parameter settings can affect revenues, costs and welfare of both the participants and the community as a whole. Although this does not constitute a proof, the expected behavior of the system is verified by experiment.

The fee collected by the community operator on each trade has a significant impact on the community. In particular, the operator fee can be increased up to the point where no exchange is worthwhile within the community. Notice that, the community can be regarded as an hedging tool, where entities can trade among themselves as long as the internal community prices are more favorable than the grid prices.

The storage device has a considerable impact on the welfare of the community, however this effect becomes less evident as the storage capacity increases.

The reported sensitivity analysis shows that the community microgrid market model proposed in [7] is a viable framework for structuring the internal market of a community microgrid, provided that appropriate values of the main parameters are selected. There is however not one set of such values that can be regarded as overall optimal, as the different stakeholders are guided by different objectives. This highlights the needs for other criteria to select a good set of values overall. These criteria could include fairness notions.

Ongoing work aims at extending this evaluation to different community configurations, and to community of communities.

\section{ACKNOWLEDGMENT}

Bertrand Cornélusse thanks Nethys for its support.

\section{REFERENCES}

[1] M. Bohman and R. Andersson, "Pricing cogenerated electricity and heat in local communities," Journal of Public Economics, vol. 33, no. 3, pp. 333-356, 1987.

[2] D. Zhang, N. J. Samsatli, A. D. Hawkes, D. J. L. Brett, N. Shah, and L. G. Papageorgiou, "Fair electricity transfer price and unit capacity selection for microgrids," Energy Economics, vol. 36, pp. 581-593, 2013.

[3] F. Moret and P. Pinson, "Energy collectives: a community and fairness based approach to future electricity markets," IEEE Transactions on Power Systems, pp. 1-1, 2018.

[4] E. Mengelkamp, J. Grttner, K. Rock, S. Kessler, L. Orsini, and C. Weinhardt, "Designing microgrid energy markets: A case study: The brooklyn microgrid," Applied Energy, vol. 210, pp. $870-880,2018$. [Online]. Available: http://www.sciencedirect.com/science/article/pii/S030626191730805X

[5] Y. Parag and B. K. Sovacool, "Electricity market design for the prosumer era," Nature Energy, vol. 1, no. 4, p. 16032, 2016.

[6] N. Liu, X. Yu, C. Wang, C. Li, L. Ma, and J. Lei, "Energy-sharing model with price-based demand response for microgrids of peer-to-peer prosumers," IEEE Transactions on Power Systems, vol. 32, no. 5, pp. 3569-3583, Sept 2017.

[7] B. Cornélusse, I. Savelli, S. Paoletti, A. Giannitrapani, and A. Vicino, "A community microgrid architecture with an internal local market," arXiv preprint arXiv:1810.09803, 2018.

[8] J. Bard, Practical bilevel optimization: applications and algorithms. Kluwer Academic Press Dordrecht, Netherlands, 1998.

[9] B. Cornélusse, D. Ernst, L. Warichet, and W. Legros, "Efficient management of a connected microgrid in belgium," in Proceedings of the 24th International Conference on Electricity Distribution, Glasgow, 12-15 June 2017, 2017. 